\title{
Deneb's variability: a hint of a deep-lying convection zone?
}

\author{
A. Gautschy
}

ETH-Zürich, ETH-Bibliothek, Rämistrasse 101, 8092 Zürich, Switzerland

e-mail: alfred@gautschy.ch

Received 15 January 2009 / Accepted 22 February 2009

\section{ABSTRACT}

\begin{abstract}
During their post - main-sequence evolution, massive star models harbor a convection zone on top of their hydrogen burning shell. This physical property helps, under suitable circumstances, to destabilize low-degree nonradial modes that might explain the cyclic light variability of $\alpha$ Cygni variables. The results of first exploratory computations demonstrate that modes with periods ranging from about one dozen to several dozen days can be overstable in star models at about the position on the HR diagram where the prototype of the class, Deneb, is observed.
\end{abstract}

Key words. stars: supergiants - stars: oscillations - stars: evolution

\section{Introduction}

Deneb ( $\alpha \mathrm{Cyg}$ ) is the prototype of a class of photometrically and spectroscopically variable supergiants. The class as such is rather loosely defined, mostly by the low amplitude of their light variability on timescales of several days to months, together with multi-periodic variability which appears to be frequent.

Figure 1 shows the position on the Hertzsprung-Russell (HR) plane of observed variable stars, which are attributed to the class of the $\alpha$ Cyg variables or also referred to as micro-variable supergiants. The data were collected from van Genderen (1989) and Lefever et al. (2007). Overlaid on the observed $\alpha$ Cyg variables are a few evolutionary tracks of star models in the mass range $13 \leq M / M_{\odot} \leq 35$ with $Z=0.02$. Most evident is the wide range in $\log T_{\text {eff }}$ and in luminosity that is to be covered by a single pulsation-driving mechanism if all these variables stars are to be explained in the framework one physically motivated class.

The long timescales and the small amplitudes associated with the variability of $\alpha$ Cyg variables leave much observational work to be done to solidly characterize the nature of variability of most of the members of this class (also of the very bright ones). Even for the prototype itself, the modal content of its variability is not reliably known. Radial-velocity observations of Deneb, obtained by Paddock in the late 1920s and the 1930s at Lick Observatory, were thoroughly analyzed by Lucy (1976). From the spectroscopic data, periods ranging roughly from 10 to 100 days were deduced and considered responsible for the cyclic variability of Deneb. The multiperiodic variability was attributed to stellar oscillations, a characteristic that also explicitly entered the definition of the class of $\alpha$ Cyg variables. Modern, highresolution, dynamical spectral monitoring of Deneb's $\mathrm{H} \alpha$ line was reported e.g. by Kaufer et al. (1996). The long dominant periods of the equivalent-width variations of $\mathrm{H} \alpha$ led Kaufer et al. (1996) to connect the source of its variability to the star's rotation with further modulation by stellar wind variability. On the other hand, the variability of the weaker lines (on timescales that differ from those of the strong lines) is still thought to be attributable to stellar oscillations.

Long-term photometric monitoring of Deneb remains rare to this day. Early on, Fath (1935) showed that the photometric

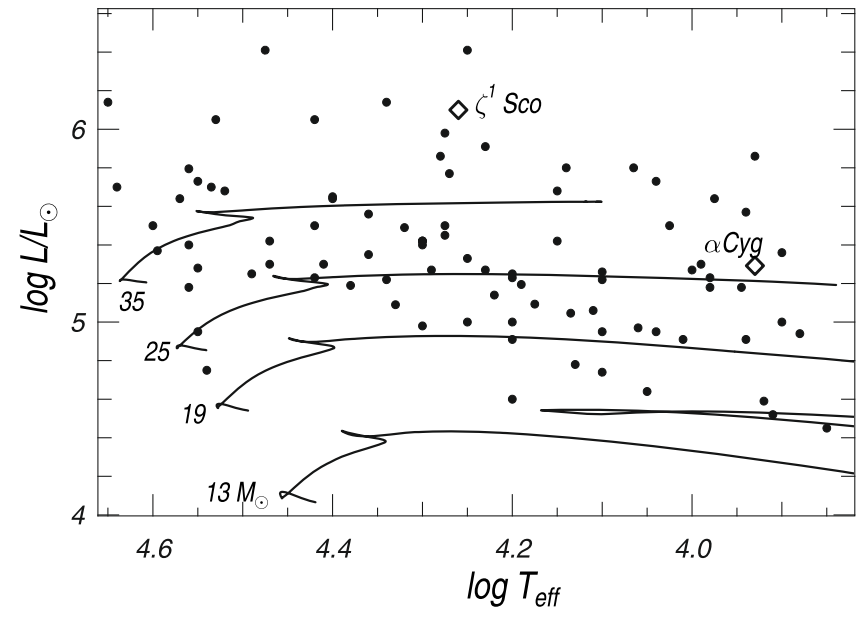

Fig. 1. HR diagram with the evolutionary tracks of numerous massive stars. The dots indicate the positions of observed $\alpha$ Cyg variables and micro-variable supergiants on the HR plane. The positions of the prototype, $\alpha$ Cygni itself and of $\zeta^{1}$ Sco, which are particularly referred to in the text, are shown by diamonds.

variability of Deneb, which he observed simultaneously with Paddock who took the spectra which were analyzed by Lucy (1976), was correlated with its radial-velocity variation. Only the data that was studied in Richardson (2006) and that spanned five years of high-resolution spectroscopy and Strömgren photometry was, to the author's knowledge, the next more comprehensive photometric monitoring of Deneb. The very regular photometric variability shown in the Richardson (2006) data is attributable to a few discrete modes with periods in the range of about 10 to 30 days. The frequencies extracted in the time-series analyses do not seem, however, to be stable across the observing seasons; whether this is an inherent variability property or an artefact due to observational biases remains unclear.

If pulsations are to be invoked as the cause of instability of the $\alpha$ Cyg variables, the width in $T_{\text {eff }}$ over which these variables are observed poses considerable problems. As of now, pulsational driving was always found to be confined to narrow 
temperature domains which explain the various instability strips across the HR diagram. Therefore, to resolve the width problem of the instability domain, strange modes were one attempt to explain $\alpha$ Cyg variability in the past (Gautschy 1992). Strange modes were successful to explain the variability of $\mathrm{R} \mathrm{CrB}$ and helium stars over their broad effective-temperature range. A prerequisite for the instability to set in is a sufficiently large ratio of $L / M$ which is indeed realized in many $\mathrm{R} \mathrm{CrB}$ and extreme helium stars. Applied to Deneb, however, the reduction of mass which was necessary to eventually arrive at overstable radial strange modes was too dramatic to be realistic. For nonradial strange modes, Glatzel \& Mehren (1996) found some instabilities of very high spherical degrees $(50<\ell<100)$ in Deneb-like $20 M_{\odot}$ models. Such instabilities cannot, however, account for Deneb's photometric variability. Therefore, as of now a strangemode explanation of $\alpha$ Cyg variability, at least for Deneb and comparable stars, does not seem to be likely.

Recently, Saio et al. (2006) found that the long-period oscillations observed in HD 163899 can be understood as overstable g-modes which are trapped in the envelope above a convection zone which overlies the hydrogen-burning shell (referred to as supra $\mathrm{H}$-shell $\mathrm{CZ}$ in the following). The stars with slow pulsations which are excited by the trapping mechanism just mentioned were referred to as SPBsg - slowly pulsating B supergiant - variables. In this report, we expand on the idea of nonradial modes being potentially trapped above the supra $\mathrm{H}$-shell $\mathrm{CZ}$ found in massive stars during their post - mainsequence phase of evolution and the possibility of their becoming overstable. We study the properties of overstable low-degree modes and their relevance for the $\alpha$ Cyg phenomenon. Our attention is focused on dipole and quadrupole modes which are potentially the most important contributors to photometric variability. Furthermore, we restricted the stability analyses to star models between the end of the main-sequence S-bend and the early core helium-burning phase of their evolution for 19 and $25 M_{\odot}$; masses which are currently thought to be reasonable limiting guesses for Deneb. The encountered instability properties of the low-degree nonradial modes in Deneb-like models are eventually used to speculate about the class of $\alpha$ Cyg variables as a whole.

\section{Stellar modeling and star models}

The stellar models referred to in the following were computed with a standard Henyey-type evolution code neglecting rotation. Opacities were interpolated in OPAL96 (Iglesias \& Rogers 1996) tables using the code made accessible by Boothroyd ${ }^{1}$. Convective mixing was treated via a diffusion equation. For the choice of the diffusion coefficient we resorted to the form put forth by Spruit (1990) which also models semiconvective mixing. We adopted an efficiency parameter which led to a quick (as compared with evolutionary changes) complete mixing also in regions with molecular-weight gradients.

Here, we are primarily interested in the pulsational behavior of Deneb; its position on the HR plane is currently estimated as $\log T_{\text {eff }}=3.93$ and $\log L / L_{\odot}=5.29$ (Schiller \& Przybilla 2008). In this exploratory study, we focus our attention on the nonradial stability properties of stellar models with $25 M_{\odot}$. Following (Schiller \& Przybilla 2008) who estimated the lower mass limit of Deneb to be about $19 M_{\odot}$, we add stability computations of evolutionary post-main sequence models with $19 M_{\odot}$ for comparison. Not only the nonradial stability properties of the early

\footnotetext{
${ }^{1}$ http://www.cita.utoronto.ca/ boothroy/kappa.html
}

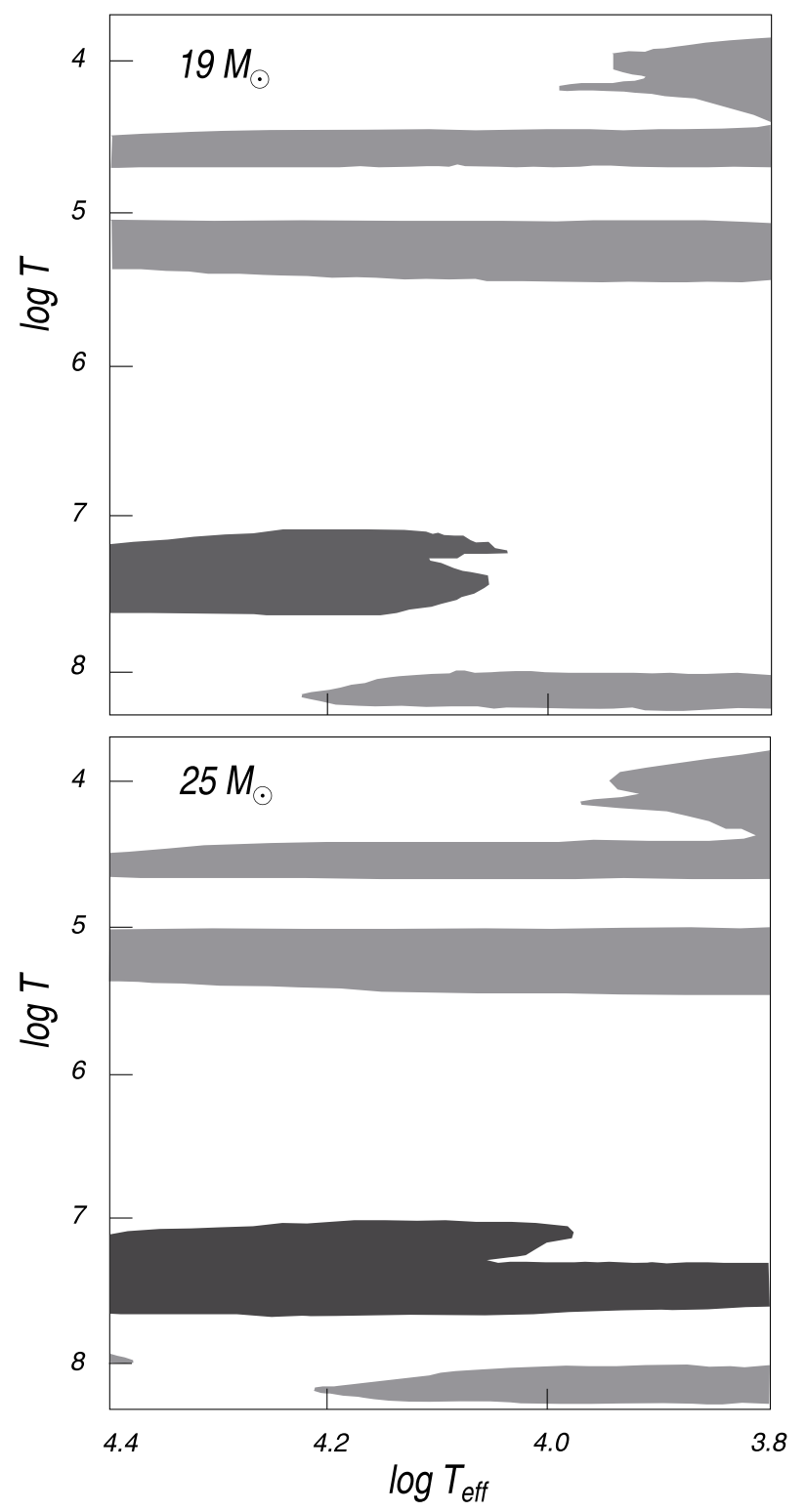

Fig. 2. Rendered positions in temperature of the convection zones of the $19 M_{\odot}$ and $25 M_{\odot}$ star models, respectively, both evolving off the main sequence (after having passed through the S-bend) and into core helium burning. The convection zone colored in dark gray which overlies the hydrogen-burning shell is referred to as supra $\mathrm{H}$-shell $\mathrm{CZ}$ in the text.

core helium burning phase, which is pertinent for a star like Deneb, is studied; the nonradial analyses are extended across the whole higher effective-temperature part of the post - mainsequence evolutionary phase to understand the connection with the SPBsg variables.

Figure 2 displays the location of the various convection zones in 19 and $25 M_{\odot}$ star models once they passed through the main-sequence S-bend on the HR plane. The ordinate, as the spatial coordinate measures the temperature. The abscissa, indicating the evolutionary phase, measures $\log T_{\text {eff }}$ for ease of comparison with the position on the HR diagram. At $\log T_{\text {eff }}=$ 4.4 , the $\mathrm{He}^{+}$partial ionization inflicts the outermost convection zone in these hot models. Only after the star cools below about $\log T_{\text {eff }}=3.9$, the increase of the opacity due to hydrogen and helium partial ionization adds two new convection layers which are initially separated but later merge as the star cools further and eventually even merge with the $\mathrm{He}^{+}$partial-ionization 


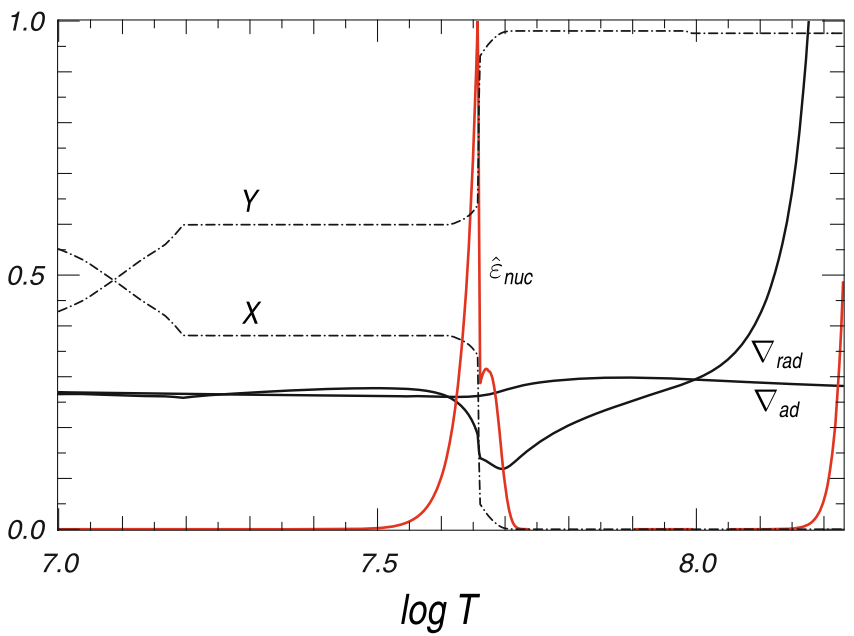

Fig. 3. Spatial variation of selected physical quantities in the deep stellar interior of the $25 M_{\odot}$ model situated at $\log T_{\text {eff }}=3.894$. The labels of the physical quantities have their canonical meanings; $\hat{\varepsilon}_{\text {nuc }}$ refers to the nuclear energy generation rate divided by its maximum value.

convection zone. At a depth of about $\log T \approx 5$, the Z-bump of the OPAL opacities enforces the next convection zone which persists from the hottest to the coolest models studied here. The convection zone highlighted with a darker gray is the one which is of particular interest: it is the convection zone which develops on top of the hydrogen-burning shell, i.e. this convection zone shows up only after the end of the main-sequence phase of the star. In the case of the $19 M_{\odot}$ model star, the supra $\mathrm{H}$-shell $\mathrm{CZ}$ vanishes at about $\log T_{\text {eff }}=4.05$ so that the potential trapping opportunity is absent for stars as cool as Deneb. On the other hand, the $25 M_{\odot}$ model stars' supra H-shell CZ persists to the coolest models studied here. The onset of core helium burning is visible indirectly in Fig. 2: at about $\log T_{\text {eff }}=4.2$ and at temperatures exceeding $10^{8} \mathrm{~K}$, a convective core develops once central helium burning gets sufficiently strong. Finally, it is informative to realize that in the $25 M_{\odot}$ case at about $\log T_{\text {eff }}=4.4$ and $\log T=8$ the end of the initial convective core can still be seen as central hydrogen burning eventually extinguishes.

Figure 3 displays selected representative physical quantities in the deep interior of the coolest $25 M_{\odot}$ model, at $\log T_{\text {eff }}=$ 3.894 , whose stability properties were studied. The dash-dotted lines show the spatial variation of the hydrogen $(\mathrm{X})$ and helium (Y) abundance. The abundance plateau out to about $\log T=$ 7.2 is a leftover of the maximum extension of the supra H-shell CZ. The sharp drop in $\mathrm{X}$ at around $\log T=7.65$ indicates the position of the H-burning shell whose normalized energy generation rate $\left(\hat{\varepsilon}_{\text {nuc }}\right)$ is plotted as the red (or gray) line. Even for the coolest model, the energy generation rate of the hydrogen burning shell exceeds that of central helium burning. The extensions of convection zones can be deduced from the relative runs of $\nabla_{\mathrm{ad}}$ and $\nabla_{\text {rad }}$.

Nonradial, nonadiabatic stability analyses on dipole and quadrupole modes were performed on the 19 and $25 M_{\odot}$ star models in the range $4.45>\log T_{\text {eff }}>3.89$ using the Riccati code which was described in e.g. Gautschy et al. (1996). On the surface of the star models, running-wave boundary conditions were adopted so that the loss of wave energy could be dealt with in the case of those eigensolutions for which no sufficiently reflecting surface layers exist. The critical frequencies delineating the propagation regions of $\ell=1 \mathrm{p}$ - and g-modes of the coolest $25 M_{\odot}$ model (at $\left.\log T_{\text {eff }}=3.894\right)$ are displayed in Fig. 4 . The

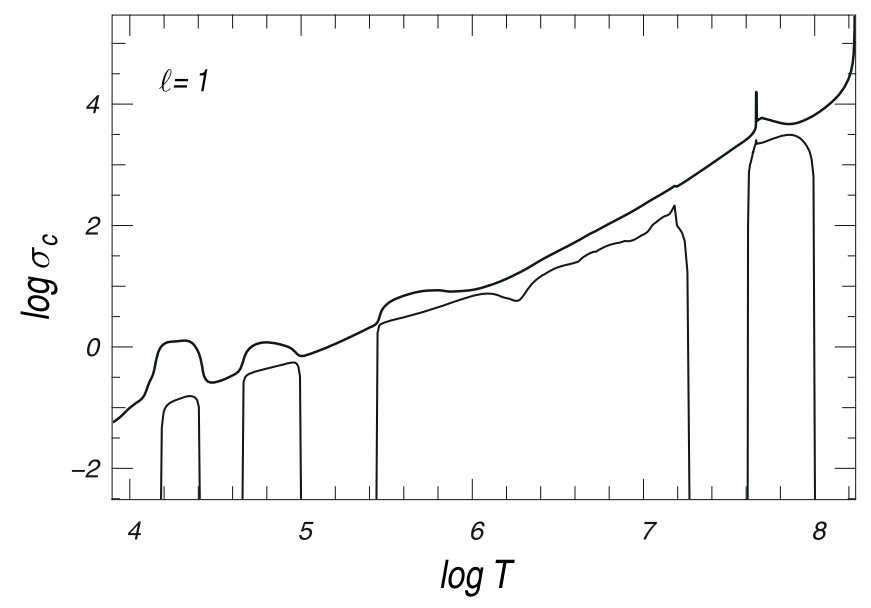

Fig. 4. Spatial variation of the logarithm of the critical frequencies of dipole modes of the $25 M_{\odot}$ model at $\log T_{\mathrm{eff}}=3.894$; the behavior is representative for star models with a supra H-shell CZ. The upper, thicker line indicates the lower bound for $\mathrm{p}$-modes to propagate. The thinner line measures the upper frequency bound for the propagation of g-modes.

normalized frequencies $\sigma$ are measured in units of $\sqrt{3 G M_{*} / R_{*}^{3}}$. The periods which are of interest in a study of $\alpha$ Cyg type variability fall roughly into the range $-1<\log \sigma_{\mathrm{R}}<0$. Hence, according to Fig. 4, the modes in which we are interested behave superficially like p-modes and deeper in the star they are spatially rapidly oscillating g-modes. As mentioned before, the stellar surface is not fully reflecting the modes of interest.

\section{Results}

Stability analyses of radial pulsation modes revealed no overstable radial modes in the period range $2<P / d<45$ for the 19 and $25 M_{\odot}$ star models. From the cyclical variation of frequency separations of neighboring radial orders, we concluded that strange modes are present, at least in the $25 M_{\odot}$ sequence, but they interact with the regular pulsation spectrum only via avoided crossings. Strange modes are therefore of no immediate relevance for $\alpha$ Cyg's variability, at least for canonical evolutionary masses.

Figure 5 displays the results from the nonradial stability computations for dipole and quadrupole modes on the 19 and the $25 M_{\odot}$ models, respectively. For reference, the diagram shows also the region of interest for Deneb: the box drawn with a heavy full line in the lower right of the panels is defined by the uncertainties in $T_{\text {eff }}$ from the spectroscopic study of Schiller \& Przybilla (2008) and by frequencies listed as having been present in one or the other observing season analyzed in Richardson (2006).

\subsection{The $19 M_{\odot}$ Sequence}

The top panel of Fig. 5 contains the frequencies, measured in units of cycles per day (c/d), of only the overstable dipole (open circles) and quadrupole modes (filled squares). For both spherical degrees, a clearly defined instability domain is encountered at high effective temperatures. For $\ell=1$, the instability region extends from $\log T_{\text {eff }}=4.356$ to $\log T_{\text {eff }}=4.150$ with frequencies ranging from 0.255 to $0.051 \mathrm{c} / \mathrm{d}$. Up to about two dozen modes can be encountered overstable at a given evolutionary stage. As mode trapping, which is discussed in detail in Sect. 3.2, 


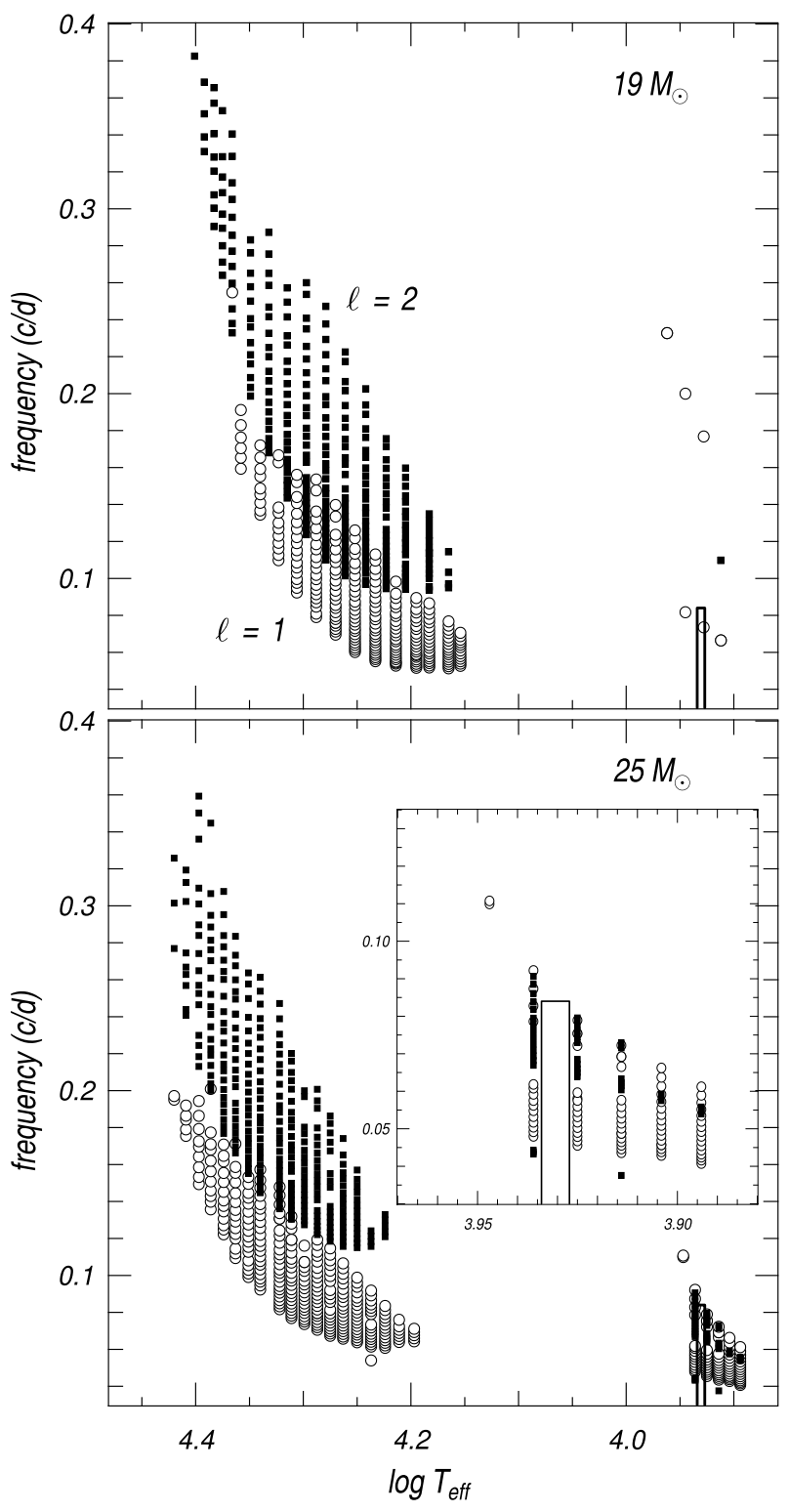

Fig. 5. Modal diagram containing the frequencies, measured in cycles per day (c/d), of overstable dipole (open circles) and quadrupole (filled squares) modes of the two exploratory star model sequences described in the text. The box in the lower right of the panels frames the region of relevance for Deneb as defined by its observations.

at the supra $\mathrm{H}$-shell $\mathrm{CZ}$ prevails, selected modes can happen to be stable in the neighborhood of otherwise overstable modes and vice versa; this effect is evident in particular close to the instability edges. Therefore, the boundaries of the instability regions look ragged. For $\ell=1$, radial orders $k$ between about 70 and 100 turn overstable.

For quadrupole modes, the instability region extends from about $\log T_{\text {eff }}=4.402$ to $\log T_{\text {eff }}=4.165$; i.e. blue and red edges of dipole and quadrupole modes are roughly the same. However, the frequencies of the quadrupole modes, lying between 0.38 and $0.094 \mathrm{c} / \mathrm{d}$, are mostly higher than those of the dipole modes at the same effective temperature; the frequency overlap is only small. The radial orders of the overstable quadrupole modes are in the range $100 \lesssim k \lesssim 130$.

At about $\log T_{\text {eff }}=4.05$, the supra H-shell CZ disappears. At lower effective temperatures - where no deep lying convective shell exists anymore - we found only two sets of weakly overstable dipole modes. The two branches seen on the right of the top panel of Fig. 5 are not made up by modes of the same radial order: in the higher-frequency branch, the radial order changes from 43 to 66; the lower frequency branch, on the other hand, is made up of modes between $k=104$ and $k=117$. In the case of quadrupole modes, only one weakly overstable mode was found with a frequency intermediate between the two dipole branches for one evolutionary model at $\log T_{\mathrm{eff}}=3.912$. The marginal instability found between $\log T_{\text {eff }}=3.9$ and 3.95 leads in any case to a much simpler variability signature of a star than what can be expected for the hotter models; at most two simultaneously overstable modes are encountered at a given evolutionary stage at these low effective temperatures.

\subsection{The $25 M_{\odot}$ Sequence}

The bottom panel of Fig. 5 displays the overstable dipole and quadrupole modes during the post - main-sequence and early core helium-burning evolution of the $25 M_{\odot}$ model.

As for the $19 M_{\odot}$ sequence, a hot instability region lies between about $\log T_{\mathrm{eff}}=4.42$ and $\log T_{\mathrm{eff}}=4.23$ for $\ell=2$ and about $\log T_{\text {eff }}=4.2$ for $\ell=1$, respectively. The frequencies of the overstable dipole modes range from about 0.054 to $0.2 \mathrm{c} / \mathrm{d}$. The overstable quadrupole modes, whose eigenfrequencies overlap only marginally with the corresponding dipole frequencies, range from about 0.11 close to the red edge to about $0.36 \mathrm{c} / \mathrm{d}$ close to the blue edge of the instability region.

Again, as in the case of the $19 M_{\odot}$ models, numerous modes were found overstable simultaneously at a given evolutionary epoch; the maximum number encountered for dipole modes was 25 at $\log T_{\text {eff }}=4.32$ and 26 for quadrupole modes at $\log T_{\text {eff }}=$ 4.34. The corresponding average radial orders of the overstable dipole modes lie around $k=210$ and around $k=170$ for the quadrupole modes.

In the range $4.2<\log T_{\text {eff }}<3.95$ of Fig. 5, no overstable dipole or quadrupole modes were found. However, in contrast to the $19 M_{\odot}$ sequence - and more importantly for Deneb - an additional instability region showed up with a blue edge, for both $\ell=1$ and $\ell=2$, at $\log T_{\text {eff }}=3.94$. To ease the discussion, this cooler instability domain is zoomed-in to and shown as an inset in the lower panel of Fig. 5. As mentioned earlier, the rectangular box outlines the observed constraints of Deneb. The red edge of the instability domain was not found in our model series which we stopped at $\log T_{\text {eff }}=3.894$. Most evidently, the instability region of the dipole modes appeared forked with the two separated frequency ranges which merge at about $\log T_{\text {eff }}=3.9$. The same was found, but less expressed, for quadrupole modes too.

For the cool instability region, the eigenfrequency behavior as a function of oscillation frequency of dipole and quadrupole modes are representatively discussed for the coolest model of the $25 M_{\odot}$ sequence (at $\left.\log T_{\text {eff }}=3.894\right)$. Figure 6 shows the period separation $P_{k+1}-P_{k}$ versus frequency $f_{k}$ (top panel) and the imaginary part of the eigenfrequency $\sigma_{\mathrm{I}}$ vs. $f_{k}$ (bottom panel) of dipole and quadrupole modes. Hence, the overstable modes (plotted with larger gray symbols) in Fig. 6 correspond to the rightmost data points in Fig. 5. The period separations of the two studied spherical degrees show cyclical depressions which correspond to local extrema in the damping rates $\sigma_{\mathrm{I}}$. For frequencies with $f>0.062 \mathrm{c} / \mathrm{d}$, these extrema correspond to locally enhanced damping rates; at lower frequencies the minima of the period separations were associated with minima in the imaginary parts of the eigenfrequencies. In the most favorable cases these locally reduced damping rates were sufficiently strong to make the corresponding modes pulsationally overstable; this is 

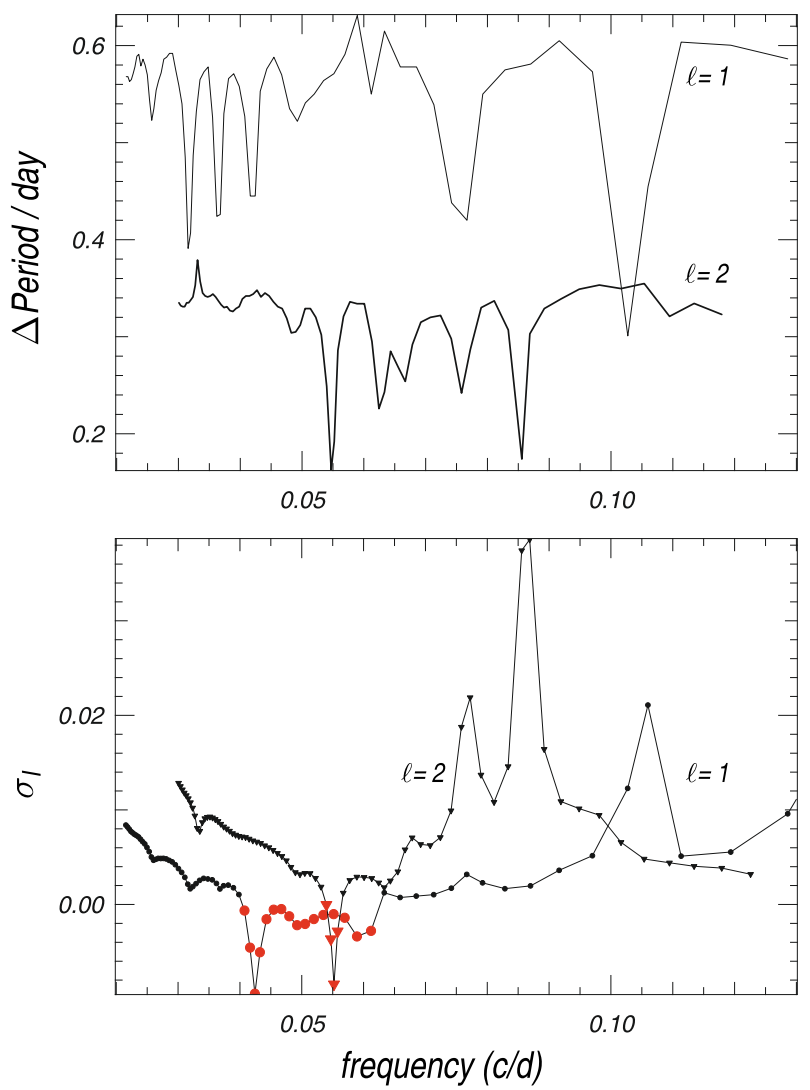

Fig. 6. Variation as a function of frequency of the period spacing of two neighboring modes (top panel) and of the damping rates (the imaginary part of the eigenvalue) of dipole and quadrupole modes (bottom panel) for the coolest $25 M_{\odot}$ star model at $\log T_{\text {eff }}=3.894$.

particularly well expressed for $\ell=2$ around $f=0.055 \mathrm{c} / \mathrm{d}$. On the other hand, for $\ell=1$, sharply localized reduced damping rates are superimposed on a broader trough in which even untrapped modes with $0.046<f<0.063 \mathrm{c} / \mathrm{d}$ happen to be overstable. This combination of mechanisms is the basis of the forking of the instability region which is visible in the lower-panel inset of Fig. 5.

\section{Discussion}

The discussion section starts with representative work integrals for the $19 M_{\odot}$ sequence to elucidate the instability mechanism of the hot instability region $\left(4.2 \lessgtr \log T_{\text {eff }} \lesssim 4.4\right)$; all essential points considered can be translated directly to the $25 M_{\odot}$ model series. For dipole and quadrupole modes, pulsation driving occurs across the Z-bump $(5.2<\log T<5.4)$. Figure 7 shows the total work integrals, measured in units of $10^{47}$, of the $\ell=1$ modes with $k=84\left(f=0.066 \mathrm{c} / \mathrm{d}, \tau_{\mathrm{e}}=7.1 \times 10^{4} \mathrm{~d}\right)$ and $k=90\left(f=0.059 \mathrm{c} / \mathrm{d}, \tau_{\mathrm{e}}=1.8 \times 10^{5} \mathrm{~d}\right)$ at $\log T_{\text {eff }}=4.183$; the e-folding time of an instability, measured in days, is stated as $\tau_{\mathrm{e}}$. At $\log T \gtrsim 5.4$, the modes are always locally damped; dissipation continues to about $\log T=7.0$. As long as the supra $\mathrm{H}$-shell $\mathrm{CZ}$ was present, no significant dissipation was ever found at temperature exceeding $10^{7} \mathrm{~K}$. Even though the $k=84$ mode has noticeable displacement and temperature-perturbation amplitudes in $7.4 \lesssim \log T \lesssim 7.6-$ which is in contrast to the $k=90$ mode and its confinement to $\log T<7.3$ - this has no influence on the work integral, the variation there is adiabatic to a high degree.

All in all, mode excitation physics, the size, and the form of the instability region of the hotter instability region seen in Fig. 5

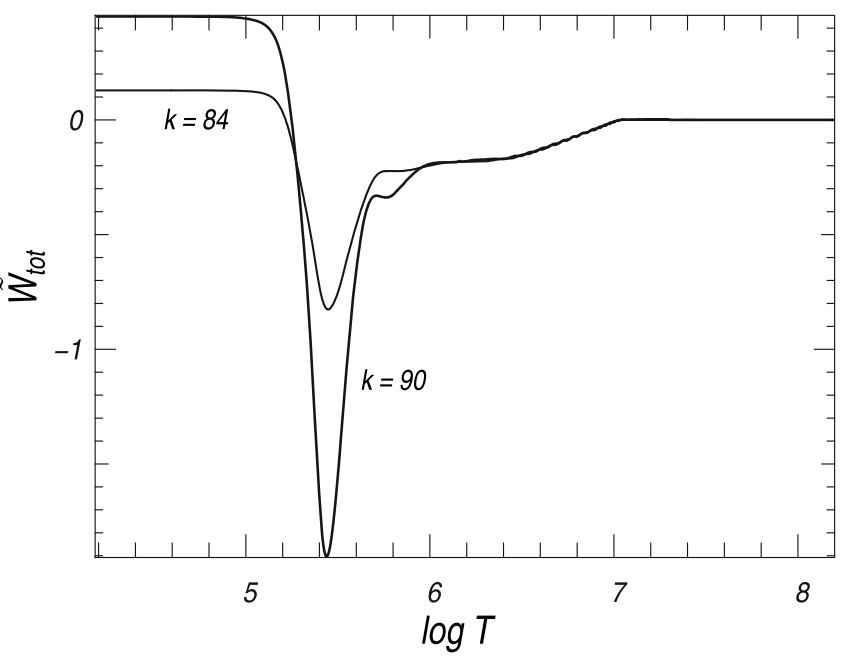

Fig. 7. Total work curves for two representative $\ell=1$ modes of the $19 M_{\odot}$ sequence at $\log T_{\text {eff }}=4.183$. The panel shows, collected in one graph, the total work, $\tilde{W}_{\text {tot }}$ which is arbitrarily divided by $10^{47}$, as a function of $\log T$ for the modes with radial orders $k=84$ and $k=90$.

Table 1. Properties of selected dipole modes for the coolest model of the $25 M_{\odot}$ sequence. The radial order is given as $k$, the ratio of superficial horizontal to radial displacement is $\xi_{\mathrm{h}} / \xi_{\mathrm{rsurf}}$, and $\eta \equiv-\sigma_{\mathrm{I}} / \sigma_{\mathrm{R}}$.

\begin{tabular}{cccccr}
\hline \hline$k$ & $P / d$ & $\left|\xi_{\mathrm{h}} / \xi_{\mathrm{r}}\right|_{\text {surf }}$ & $\max \left|\xi_{\mathrm{h}} / r\right|$ & $\eta$ & Character \\
\hline 72 & 21.42 & 0.623 & 78.3 & $2.15(-4)$ & untrapped \\
77 & 23.57 & 0.055 & 7.3 & $4.56(-3)$ & trapped \\
152 & 46.4 & 0.500 & 40.9 & $-7.98(-3)$ & untrapped \\
\hline
\end{tabular}

agree all well with the results discussed in the discovery paper of the SPBsg variability by Saio et al. (2006).

The overstability of the few modes, actually of two branches of overstable modes of varying radial order, in the narrow range $3.9<\log T_{\text {eff }}<3.95$ (on the right of the top panel of Fig. 5) can be attributed to mode trapping as well. Figure 4 shows the essentials even though it belongs to a $25 M_{\odot}$ model and still has an active supra $\mathrm{H}$-shell $\mathrm{CZ}$; the important part which applies also to the $19 M_{\odot}$ models at $\log T_{\text {eff }}<4.0$ are the critical frequencies at around the Z-bump convection zone (spanning $5.0 \lesssim \log T \lesssim 5.4$ ). The oscillation frequencies, $\sigma_{\mathrm{R}}$, of the overstable modes of the cool $19 M_{\odot}$ models, normalized in the same way as $\sigma_{\mathrm{C}}$, lie between two and four. Hence, above the Z-bump $\mathrm{CZ}$, these modes propagate as p-modes, and below they behave like g-modes. If a mode manages to place a node of the horizontal displacement close to the convective boundary at $\log T=5.4$, the eigencomponents are quenched in the deeper interior so that the dissipation, which is picked up in the regions with rapid spatial oscillation of the eigenfunctions and significant corresponding amplitudes $(7.2<\log T<7.55)$, is suppressed and pulsational driving of the $\mathrm{H} / \mathrm{He}$ partial ionization zone can turn such a mode overstable. Inspection of the eigenfunctions indeed shows maximum horizontal displacement amplitudes which are more than a thousand times smaller for trapped modes than for untrapped (damped) ones. The character of the eigenfunctions is much more reminiscent of $\mathrm{p}$-modes rather than mixed modes with g-mode behavior in the deep interior. Furthermore, the kinetic energies of the overstable, trapped modes are two to five orders of magnitude smaller than those of untrapped modes, emphasizing the trapping to the superficial regions of the star.

For the discussion of the cool instability region (at $\log T_{\text {eff }}<$ $3.95)$ of the $25 M_{\odot}$ sequence, Table 1 collects properties of 


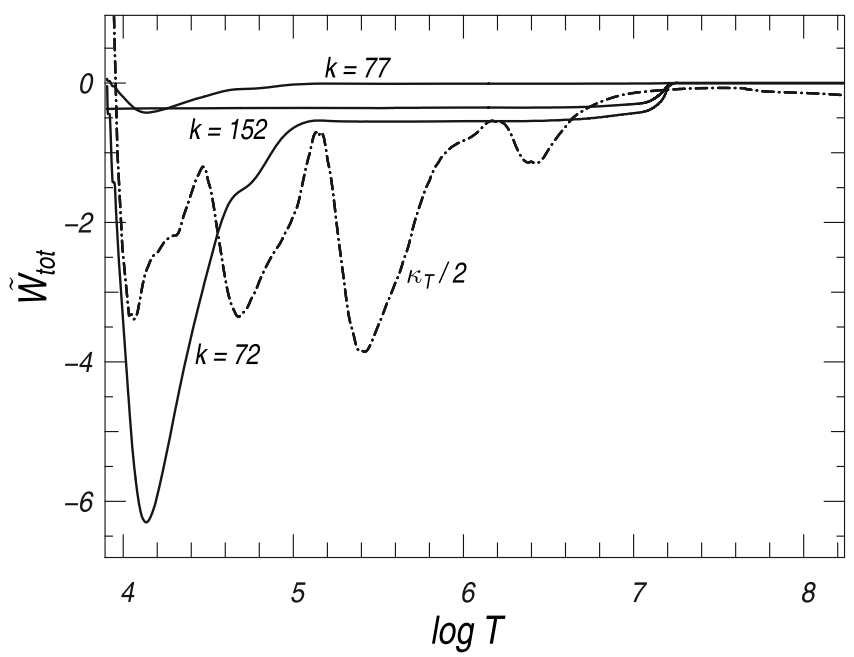

Fig. 8. Total work curves for selected, representative $\ell=1$ modes of the $25 M_{\odot}$ sequence at $\log T_{\text {eff }}=3.894$. The full lines show the total work, $\tilde{W}_{\text {tot }}$, again total work divided by $10^{47}$, as a function of $\log T$ for the modes with radial orders $k=72,77$, and 152 (which are described in Table 1). The dash-dotted line shows, for orientation, the spatial variation of $\kappa_{T} / 2$.

representative dipole modes; it includes untrapped damped modes (illustrated with the $k=152$ mode), untrapped overstable modes (represented by the $k=72$ mode), and trapped overstable modes like the $k=77$ mode. The radial order was determined by counting nodes of the real part of the displacement eigenfunction component $\xi_{\mathrm{r}} / r$. The modulus of the ratio of the real part of the superficial horizontal to radial displacement is listed under $\left|\xi_{\mathrm{h}} / \xi_{\mathrm{r}}\right|_{\text {surf }}$. The quantity $\eta$ denotes the normalized growth rate computed as $-\sigma_{\mathrm{I}} / \sigma_{\mathrm{R}}$; the exponents are given as integers in brackets. The rightmost column roughly characterizes the mode.

Total work integrals of the oscillation modes highlighted in Table 1 are presented in Fig. 8; it displays the total work divided by $10^{47}$ of overstable dipole modes with radial order $k=72,77$, and of the stable mode with $k=152$. For illustration, the spatial variation of the opacity derivative $\kappa_{T} / 2$ is added to the plot. The two untrapped modes $(k=72,152)$ pick up damping in the deep interior, above the supra $\mathrm{H}$-shell $\mathrm{CZ}$, where the eigenfunctions oscillate rapidly. The trapped, overstable mode with $k=77$ does not experience noticeable damping in the deep interior; local dissipation of pulsation energy of this mode, as well as for the $k=72$ one with comparable period, occurs in the layers with $4.2<\log T<5$.0, i.e. also across the $\mathrm{He}^{+}$-partial ionization zone. Pulsational driving, on the other hand, which eventually makes both modes $(k=72,77)$ overstable occurs all in the $\mathrm{H}-$, He-partial ionization region. On the other hand, the longer period $k=152$ mode experiences neither driving nor damping in the envelope ionization zones; its fate is determined by the damping in the deep interior.

To illustrate the spatial character of the eigenmodes, Fig. 9 shows the relative radial displacement eigenfunctions for representative modes which were picked out in Table 1. As mentioned in the discussion of the total work integrals, the trapped $k=77$ mode has a reduced amplitude in the deep interior which is compatible with the lack of damping picked up in this region of the star. As it is to be expected from the mixed-mode character of the modes, with p-mode behavior in the superficial layers and the g-like propagation region of the deeper stellar interior (roughly for $\log T>5$ ), the amplitude of horizontal displacement exceeds there the radial component considerably;

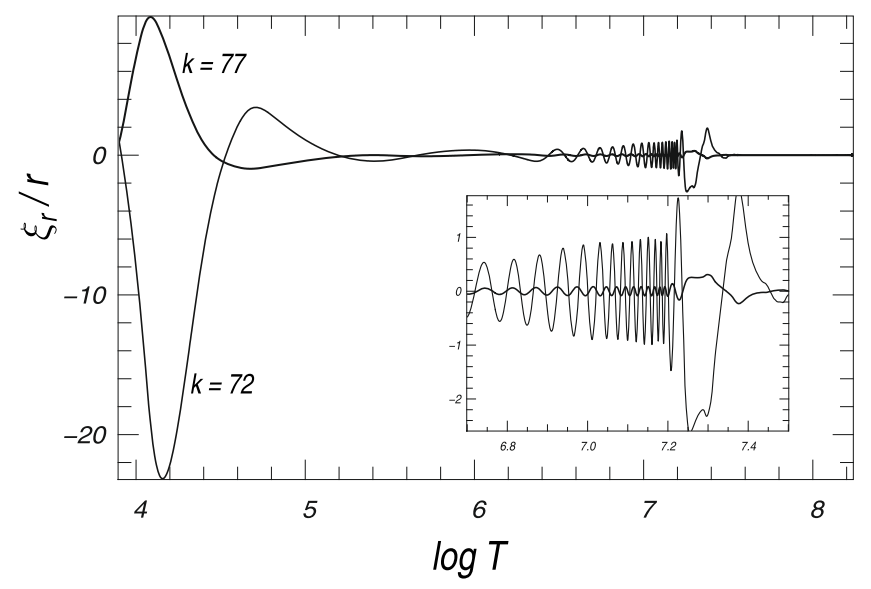

Fig. 9. Panel a): radial displacement component $\xi_{\mathrm{r}} / r$ of the eigenfunctions of two representative modes at $\log T_{\text {eff }}=3.894$ labeled with their radial orders $k$. A blow-up of the region around the supra $\mathrm{H}$-shell $\mathrm{CZ}$ is inserted.

according to Table 1 , the maximum of $\left|\xi_{\mathrm{h}} / r\right|$ reaches about 41 and 78 for the untrapped example modes but only about 7 for the trapped one. The maxima are attained in all cases near the upper edge of the supra $\mathrm{H}$-shell $\mathrm{CZ}$. Again, also the horizontal displacement reflects the trapping effect with its reduced amplitude, in particular in the deep interior.

Despite extensive searches on the complex plane, we found no evidence of nonradial pulsation modes which are excited by the action of the $\mathrm{He}^{+}$-partial ionization zone in the intermediate effective-temperature range $3.95 \lesssim \log T_{\text {eff }} \lesssim 4.15$.

Our emphasizing of overstable nonradial pulsation modes which depend on the existence of a deep-lying convection zone to explain $\alpha$ Cyg instability means that the main-sequence members of the observed $\alpha$ Cyg variables and which are assigned to the class by van Genderen (1989) should be discarded. Even if from a phenomenological point of view, these main-sequence pulsators are comparable to the Deneb-like pulsators - their pulsations must be driven by a different physical mechanism. It is not unlikely that these main-sequence pulsators can be accommodated in a high-luminosity extension of the $\beta$ Cep instability domain.

Extending the experience from our preliminary computations of Deneb's variability to the whole - loosely defined - class of the $\alpha$ Cyg variables it seems that the conjecture stated in a subordinate clause of the Glatzel \& Mehren (1996) paper might eventually be true: the $\alpha$ Cyg variables are not a homogeneous class with respect to the excitation mechanism of their variability. The observational signature looks comparable for many cyclically low-amplitude variable supergiants; however, depending on mass and evolutionary state, different physical agents excite the stellar oscillations.

As this report is only a step towards an explanation of the $\alpha$ Cyg variables rather than a final word regarding the physics of their variability, the paper closes with a theoretical and an observational plea which hopefully help to push the field ahead.

\subsection{Modeling plea}

The range of stellar masses which are to be studied in detail must be extended: stars from 15 to at least $40 M_{\odot}$ should be included in a survey of pulsation properties.

The $\alpha$ Cyg variables which still live within the mainsequence domain (see Fig. 1) and which were explicitly added 
to the class by van Genderen (1989) because of their comparable photometric variability signature do not fit into our mode trapping model. Even if their photometric variability is like that of the post main-sequence variables of the class of the $\alpha$ Cyg stars, the physical origin of their variability must be different. The "main-sequence $\alpha$ Cyg variables" fall roughly into the highluminosity extension of the $\beta$ Cep instability domain, so we expect that they should naturally fit into this class of variable stars. A definite answer can, however, only be given after detailed corresponding computations are performed.

It is still possible that, in addition to the aspects addressed above, the high-luminosity part of the $\alpha$ Cyg variability domain contains contributions from strange modes. The strange-mode aspect was not part of the current project; but it should be revisited once again in the future; at least for the most luminous $\alpha$ Cyg variables. Stars, like $\zeta^{1}$ Sco (cf. Fig. 1), with luminosities exceeding $10^{6} L_{\odot}$, have luminosity-to-mass ratios which commonly support strange modes.

\subsection{Observational plea}

Most of all, the variability properties of the prototype itself should eventually be reliably determined so that it can be referred to with confidence as a template to classify other blue to yellow supergiants and that it can serve as the basis of thorough theoretical investigations that try to pin down the physical origin of the variability.

The fact that Deneb is a first-magnitude star makes it a very easy target for high-precision photometry, but only for sufficiently small-aperture instruments; all the ongoing or planned sky-monitoring projects are of no help for such a purpose as their detectors saturate too rapidly on Deneb. Therefore, the setting-up of a long-term variability monitoring project of the brightest $\alpha$ Cyg stars in the sky (including Deneb, $\beta$ Ori, $\zeta^{1}$ Sco, V360 Nor, LR Vel) with suitable modern off-the-shelf instruments that achieve a photometric accuracy of better than 0.01 mag would be most rewarding.

If strange modes can be hold responsible for or at least contribute to the variability of high-luminosity $\alpha$ Cyg variables, then $\zeta^{1}$ Sco (cf. Fig. 1) is the bright candidate which is particularly worth monitoring. For strange-mode pulsators, the bolometric luminosity variation can be small; therefore, multicolor or even spectrophotometric observations should be helpful to distinguish between strange-mode variables and "canonical", $\kappa$-driven pulsators like Deneb itself.

Looking at the $\alpha$ Cyg variables as a class, is it possible to observationally confirm the modeling conjecture of the absence of variable supergiants in the effective-temperature range between about 9000 and $14000 \mathrm{~K}$ ?

For a fixed choice of mixing efficiency, the life-time of the supra H-shell $\mathrm{CZ}$ was found to be a function of the heavy-element abundance $Z$. Evolutionary computations with an SMC-like choice of $Z=0.004$ revealed the red edge of the supra $\mathrm{H}$-shell CZ at $\log T_{\text {eff }}=4.03$ (as compared with $\log T_{\text {eff }}<3.8$ in Fig. 2 for $Z=0.02$ ). Hence, if the supra $\mathrm{H}$-shell $\mathrm{CZ}$ is indeed instrumental for the $\alpha \mathrm{Cyg}$ - type variability of massive stars as suggested in this paper, then we predict that the Galaxy contains considerably cooler variables of this type than for example the SMC; or at the very least the SMC should harbor cool supergiant variables with simple (as compared to Deneb) variability signatures if mode trapping at the Z-bump CZ persists.

Acknowledgements. This research has made use of NASA's Astrophysical Data System Abstract Service. Sporadic trapping between Fendant and Chèvre in $\mathrm{H}$. Harzenmoser's kitchen contributed considerably to advance the conceptual understanding of $\alpha$ Cyg variables. Hideyuki Saio's pertinent comments and discussions helped to raise the quality of the research.

\section{References}

Fath, E. 1935, Lick Obs. Bull., 17, 115

Gautschy, A. 1992, MNRAS, 259, 82

Gautschy, A., Ludwig, H.-L., \& Freytag, B. 1996, A\&A, 311, 493

Glatzel, W., \& Mehren, S. 1996, MNRAS, 282, 1470

Iglesias, C., \& Rogers, F. 1996, ApJ, 464, 943

Kaufer, A., Stahl, O., Wolf, B., et al. 1996, A\&A, 305, 887

Lefever, K., Puls, J., \& Aerts, C. 2007, A\&A, 463, 1093

Lucy, L. 1976, ApJ, 206, 499

Richardson, N. 2006, Master's thesis, University of Toledo

Saio, H., Kuschnig, R., Gautschy, A., et al. 2006, ApJ, 650, 1111

Schiller, F., \& Przybilla, N. 2008, A\&A, 479, 849

Spruit, H. 1990, A\&A, 239, 443

van Genderen, A. 1989, A\&A, 208, 135 